\title{
Patellofemoral evaluation with magnetic resonance imaging in 51 knees of asymptomatic subjects
}

\author{
L. Nicolaas $\cdot$ S. Tigchelaar $\cdot$ S. Koëter
}

Received: 24 January 2010/ Accepted: 31 March 2011/Published online: 30 April 2011

(C) The Author(s) 2011. This article is published with open access at Springerlink.com

\begin{abstract}
Purpose The objective of this study is to evaluate patellofemoral joint imaging on magnetic resonance imaging (MRI) in asymptomatic subjects to assess normal values and to test statistical correlation and reliability of MRI scan.

Methods An analysis of 51 standard MRI examinations was performed. Sulcus angle (SA), patellar axis (PA), lateral patellofemoral angle (LPFA), and lateral patellofemoral length (LPL) were measured. None of the patients suffered from patellofemoral complaints. Patients with patella alta and significant hydrops were excluded. The measurements were assessed with a 2-week interval by two raters under blinded conditions. Statistical analysis was applied by an independent analyst.

Results The mean SA referenced $142.4 \pm 6.9^{\circ}$, PA $5.3 \pm 3.8^{\circ}$, LPFA $13 \pm 4.4^{\circ}$, and LPL $0.8 \pm 2.9 \mathrm{~mm}$. Inter-observer variability showed high correlation for LPL and PA, as the repeatability coefficient was high (LPL; 1.49 (LN), 5.7 (ST) and PA; 4.1 (LN), 5.8 (ST). Also, intra-observer variability showed good correlation for LPL and PA.

Conclusion The results represent patellofemoral values in the normal population. They indicate that MRI is a reliable imaging technique to determine lateral patellofemoral length and patellar axis. Lateral patellofemoral angle and sulcus angle showed a poor correlation and should not be used for decision making.
\end{abstract}

L. Nicolaas · S. Tigchelaar

Department of Orthopaedic Surgery, Radboud University

Nijmegen Medical Centre, Nijmegen, The Netherlands

S. Koëter ( $\square)$

Department of Orthopaedic Surgery, Canisius Wilhelmina Hospital, PO box 9015, 6500 GS Nijmegen, The Netherlands e-mail: S.Koeter@cwz.nl
Level of evidence Development of diagnostic criteria in a consecutive series of patients and a universally applied "gold" standard, Level II.

Keywords MRI - Patella dislocations - MPFL rupture Trochlea dysplasia $\cdot$ Observer variability $\cdot$ Limits of agreement

\section{Introduction}

Patellofemoral instability is a disorder which is caused by a number of anatomic abnormalities. In order to qualify and quantify these abnormalities, radiologic imaging techniques are key to the evaluation of the patellofemoral joint. Plain radiography is the most common diagnostic imaging tool and is the least costly. It is usually supplemented by a computed tomography (CT), because the CT is 1.5 times more sensitive in detecting anomalies that cause patellar instability when compared with conventional radiography [13].

Although CT scanning has been proven to be reliable in evaluation of distal patellar malalignment $[3,7,14]$, it has major drawbacks compared with magnetic resonance imaging (MRI). It does not allow for (1) imaging of the cartilage morphology. This morphology is essential for joint congruency and thus for joint stability. As it has been shown that cartilaginous morphology differs from that of the underlying bony trochlea, MRI would be the imaging technique of choice for evaluating the trochlear groove [10, $18,19]$. Besides a better evaluation of cartilaginous morphology, MRI also allows for imaging of (2) chondral lesions after patella dislocations and (3) ruptures to the MPFL [17, 20]. The incidence of chondral lesions after a patella dislocation is reported to be as high as $96 \%$ [12]. 
A fourth benefit is that MRI does not expose patients to radiation.

Four different factors have been proved to be significantly correlated with recurrent patellar instability. These factors are as follows: trochlea dysplasia, patella alta, medial patellofemoral ligament (MPFL) rupture, and an increased tibial tubercle-trochlear groove (TT-TG) distance [6]. Thus far, only patellar height $[2,11]$ and trochlear groove tibial tuberosity distance measurements have been validated for MRI scans [15]. Of the two other important factors in patellofemoral instability, i.e. trochlea dysplasia and patella tilt (as an expression of MPFL insufficience), neither reference values nor reliability is available for MRI scans. The aim of this study was to describe a reliable alternative to CT scanning because of its inherent disadvantages; therefore, we evaluated which MRI measurements can be relied on for assessing trochlea dysplasia and patella tilt. We evaluated the sulcus angle (SA), patellar axis (PA), lateral patellofemoral angle (LPFA), and lateral patellofemoral length (LPL) using cartilaginous landmarks and tested the statistical correlation and reliability.

\section{Materials and methods}

Eighty-three consecutive patients (83 knees) from the outpatient knee clinic of the Alysis Rijnstate hospital (Arnhem, the Netherlands) were evaluated for this study. All patients obtained standard MRI scanning after a traumatic event of the knee. MRI examinations were performed using an Intera release 12, 1.5 Tesla Philips MRI scanner with a slice thickness of $3 \mathrm{~mm}$. The position of the knee was in $30^{\circ}$ flexion with the quadriceps muscle relaxed.

The most common diagnoses were meniscal or anterior cruciate ligament pathologies. Thirty-two patients suspected of patella alta, fractures, or significant hydrops were excluded. Thus, the cohort reflected the normal population. An analysis of 51 standard MRI examinations of 51 consecutive patients was performed. None of these patients suffered from patellofemoral complaints. None of the MRI scans were rejected because of inadequate quality.

Measurements on axial MR images included the following parameters: The sulcus angle (SA) was measured using the chondral outline of the trochlea with the axial section at the level of the Roman arch (Fig. 1). Also at this level, we measured the patellar axis (PA) as described by Dejour et al. [6], which is the angle between the midline axial section of the patella and the line drawn parallel to the posterior femoral condyles (Fig. 2). The lateral patellofemoral length (LPL) is the distance between the most lateral part of the patella and the line drawn parallel to the lateral side of the femur condyl (Fig. 3). Lateral patellofemoral angle (LPFA), as described by Laurin et al. [10], is the

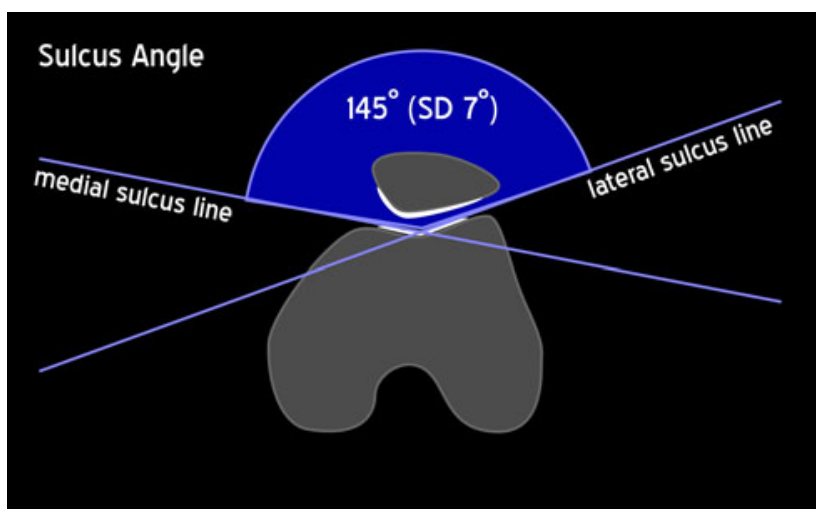

Fig. 1 Sulcus angle

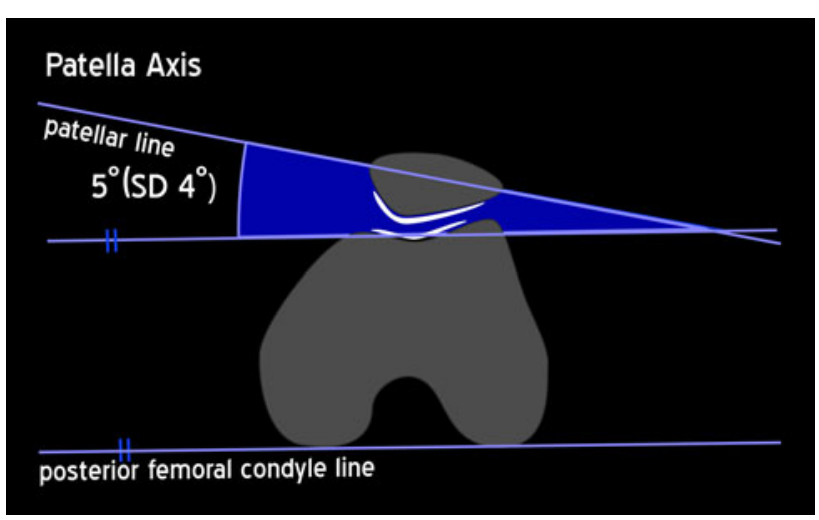

Fig. 2 Patella axis

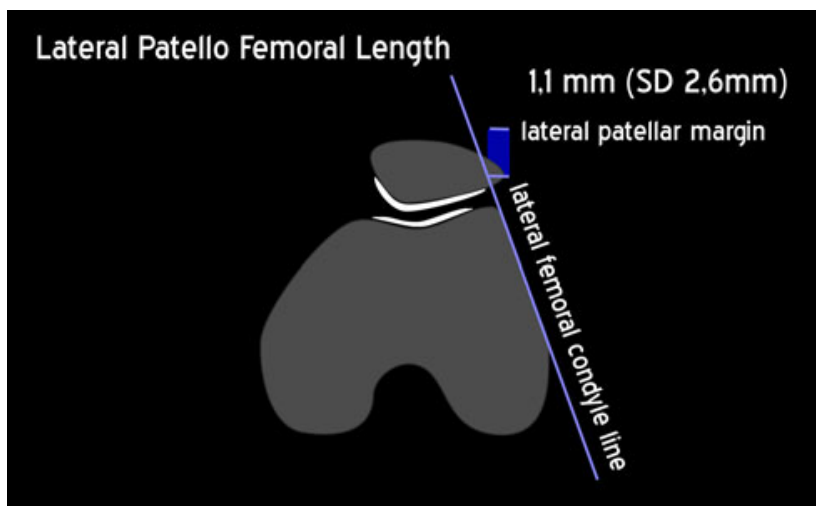

Fig. 3 Lateral patellofemoral length

angle between the line parallel to the tip of the anterior condyles and the lateral patellar facet (Fig. 4). Values were measured in degrees (SA, PA, and LPFA) and millimeters (LPL) up to one-decimal accuracy.

Two raters with different medical experiences conducted al measurements: one medical student (last year medical school) and one orthopaedic resident (third-year resident) (LN, ST). The measurements were assessed twice with a minimal interval of 6 weeks. At every assessment, 


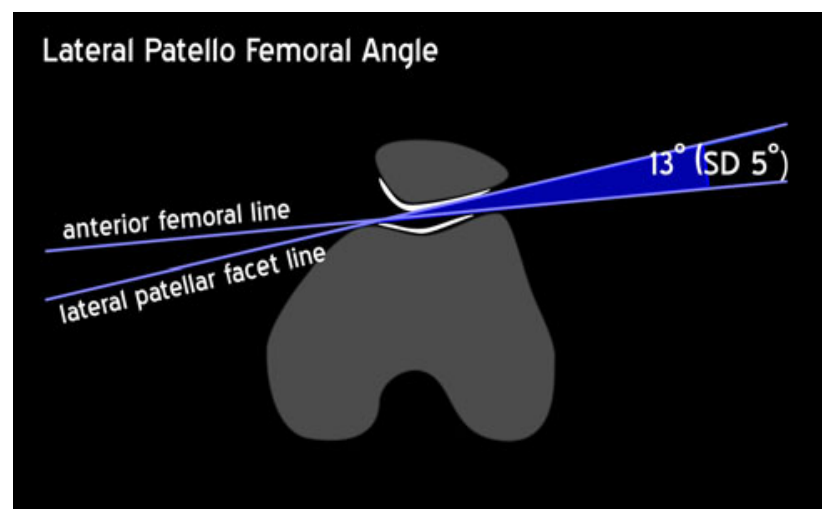

Fig. 4 Lateral patellofemoral angle

the measurements were performed twice by each observer, and the mean of those measurements was used in statistical analysis. All observations were assessed under blinded conditions [4]. The observers were not involved in the treatment of the patients.

\section{Statistical analysis}

To determine the inter- and intra-observer reliability, the coefficient of repeatability was calculated. The coefficient of repeatability is a qualitative measure of rater reproducibility and is based on the expectation that $95 \%$ of the differences between measurements for one observer and between the two observers are smaller than $1.96 \times$ standard deviation (SD) of the differences.

To determine the limits of agreement, the Bland and Altman method was used [5]. The limits of agreement, defined as the mean difference between observers $\pm 1.96 \times \mathrm{SD}$ of this mean difference, quantify the reliability of the measurement techniques as it determines the measurement error from all sources (the inter- and intravariability from the observers). Thus, the limits of agreement indicate the minimum difference between two measurements that is needed to exceed the measurement error.

\section{Results}

The mean values for SA, PA, LPL, and LPFA referenced respectively $\quad($ mean $\pm \mathrm{SD}): \quad \mathrm{SA} \quad 142.4 \pm 6.9^{\circ}, \quad \mathrm{PA}$ $5.3 \pm 3.8^{\circ}$, LPL $0.8 \pm 2.9 \mathrm{~mm}$, and LPFA $13 \pm 4.4^{\circ}$.

As shown in Table 1, the repeatability was higher in LPL and PA, as indicated by a lower repeatability coefficient. The repeatability coefficient was 1.5 (ST) and 5.7 (LN) for LPL and 4.1 (LN) and 5.8 (ST) for PA measurements. Both LPFA and SA showed higher variability between the measurements, as the repeatability coefficient
Table 1 Repeatability

\begin{tabular}{lll}
\hline & Observer 1 (LN) $^{1}$ & Observer 2 (ST) $^{1}$ \\
\hline Lateral patellofemoral angle & 6 & 10.2 \\
Sulcus angle & 5 & 9.1 \\
Patellar axis & 4.1 & 5.8 \\
Patellofemoral length & 1.5 & 5.7 \\
\hline
\end{tabular}

${ }^{1}$ Coefficient of repeatability $=1.96 \times$ standard deviation differences for 1 observer and method $=2.77 \times$ root of the mean within-subject variation

Table 2 Limits of agreement

\begin{tabular}{lll}
\hline & $\begin{array}{l}\text { Based on 1 } \\
\text { measurement }^{2}\end{array}$ & $\begin{array}{l}\text { Based on 2 } \\
\text { measurements }^{3}\end{array}$ \\
\hline Lateral patellofemoral angle & 11 & 9.6 \\
Sulcus angle & 9.3 & 9 \\
Patellar axis & 5.4 & 5.7 \\
Patellofemoral length & 5.1 & 4.6 \\
\hline
\end{tabular}

$\overline{21.96 \times \text { standard deviation differences for } 2 \text { or more observers or }}$ methods for 1 measurement

$31.96 \times$ standard deviation differences for 2 or more observers or methods corrected for multiple measurements per observer

was higher for both observers. The repeatability coefficient for LPFA was $6(\mathrm{LN})$ and $10.2(\mathrm{ST})$, and for SA 5 (LN) and 9.1 (ST). In inter-observer as well as intra-observer variability, PA and LPFA measurements represent the best results (Table 1).

The limits of agreement determine the measurement error from all sources, and it quantifies the reliability of the parameters. As shown in Table 2, the results for SA show $9.3^{\circ}$ and $9^{\circ}$ based on one and two measurements, respectively. Thus, if the SA is measured by one observer, a difference greater than $9.3^{\circ}$ is a real difference; the difference must be greater than $9^{\circ}$ to be classified as a real difference if two different observers measured the values. Thus, the actual value of SA could be up to $9.3^{\circ}$ lower or greater than the value measured, and the difference between the two measurements made by different observers must be greater than $9^{\circ}$ to be classified as being a real difference. These results of SA imply high levels of variation. The limits of agreement calculated for PA and LPL were low, being $5.4^{\circ}$ based on one and $5.7^{\circ}$ based on two measurements for PA and $5.1 \mathrm{~mm}$ and $4.6 \mathrm{~mm}$ for LPL. LPFA had the highest limits of agreement: $11^{\circ}$ and $9.6^{\circ}$, respectively.

\section{Discussion}

The most important finding of this study is that a MRI can be used for evaluating the patellofemoral joint but that not 
all measurements are equally reliable. MRI is a reliable technique to assess PA and LPL, but not for evaluating the SA and LPFA, because these measurements have a poor inter- and intra-observer reliability. This study gives an estimate of the cartilaginous normal values of SA, LPFA, PA, and LPL, and it is the first time that these normal values for MRI imaging of the patellofemoral joint were described. The clinical relevance of this study is that it proves that a MRI scan can be used for evaluating the patellofemoral joint, but not all measurements are equally reliable.

To evaluate pathologies of the patellofemoral joint on MRI, normal values are necessary to interpret abnormal findings as ill-defined values may lead to poor surgical decision making. A MRI instead of a CT scan can result in conceivable changes in treatment of patients with both primary and recurrent patellar dislocations because a MRI can show abnormalities which can be overlooked on a CT scan. Examples are a large chondral fracture (that needs operative treatment) found after a primary dislocation or a dysplastic cartilaginous femoral trochlea found in recurrent instability patients. The cartilaginous joint congruency is important because the objective of any stabilizing surgical procedure is to try to restore the joint congruency to normal, and therefore stable, values [13].

Is the MRI now shown to be reliable on evaluating the patellofemoral joint? We only looked at a limited number of measurements that we thought important. We did not evaluate all patellofemoral measurements using MRI previously described by other authors. Patella alta has been thoroughly researched by numerous authors which all came to the conclusion that MRI is a suitable imaging technique for measuring patella alta, showing good inter- and intraobserver reliability among the different methods of measuring patella alta, although the more recently introduced patellotrochlear index by Biedert et al. showed poor correlation in assessing patella alta when compared with the more conventional methods such as the Blackburne-Peel, Caton-Deschamps, and Insall Salvati ratios [2, 4, 11]. The tibial tubercle-trochlear groove distance (TT-TG) has been studied by Schoettle et al., and the bony and cartilaginous landmarks of the trochlea have been studied by Huyssteen et al. [8, 15]. Schoettle et al. [15] showed that TT-TG can be reliably determined on MRI. They found an interobserver and intra-observer variability comparable to the results in this study.

Huyssteen et al. [8] showed that cartilaginous trochlear morphology differs markedly from that of the underlying bony trochlea. They found a mean angle of the bony sulcus of $138.2^{\circ}(126-157$; SD 7.2) and a cartilaginous sulcus of $147^{\circ}$ (133-179; SD 9.5) in their control group. In this study, we now found the cartilaginous SA value to be $145.3 \pm 7.1^{\circ}$, but the measurement reliability of this angle was poor. None of the chondral landmarks of the LPFA, LPL, and PA on MRI were reported in previous studies. The LPFA was first described by Laurin et al. [10] based on conventional axial radiographs. They found normal values for LPFA to be $>0^{\circ}$. This value was later confirmed by Inoue et al. on CT scanning [9]. Alemparte et al. [1] found the LPFA on CT scanning to be $8.1 \pm 14.5^{\circ}$, which is close to our results of $13 \pm 4.4^{\circ}$ on MRI. The PA was first described by Dejour et al. [6] as measured on CT scanning. They described a PA of $10 \pm 5.8^{\circ}$ in non-symptomatic persons. More recently, Alemparte et al. [1] found a PA of $11.1 \pm 10.6^{\circ}$, also on CT scanning. Our results of $5.3 \pm 3.8^{\circ}$ as measured on MRI are remarkably smaller.

Limitations of this study consist of small population size, two raters who varied in experience, and the chosen population type. Fifty-one knees were evaluated, which makes the sample of fairly small size, but sufficient for statistical analysis. Two different raters performed the measurements in current study. Both raters varied in orthopaedic experience, which may have influenced the inter-observer variability. The results were improved at time of the second measurement assessment. This suggests a learning curve for all four measurements. On the other hand, these results indicate that even inexperienced raters can perform all measurements. This is represented in the lower numbers of repeatability coefficient for the less experienced rater, which implies that the measurement method is simple.

The current population should represent the normal population without patellofemoral complaints. Nevertheless, all patients used in this study have gone through a traumatic event. Patients with evident fractures or significant hydrops were excluded, but patients who would have had an anterior cruciate ligament (ACL) deficiency were not. As is now known, an ACL deficiency induces an increased patellar angle of approximately $3^{\circ}$ [16]. We found that the difference between measurements must be at least $5^{\circ}$ to be a real difference, so a difference of only $3^{\circ}$ may not be so relevant. The current study population was limited to Dutch subjects. This should represent a Caucasian population, but due to the anatomical nature of the study, results cannot be extrapolated to other populations.

\section{Conclusion}

The results represent patellofemoral values on MRI imaging in a normal population without patellofemoral complaints. The results indicate that MRI is a reliable imaging technique to determine patellar axis and lateral patellofemoral length. Lateral patellofemoral angle and sulcus angle showed lesser results for intra-observer correlation as well as inter-observer correlation and should not be used for 
decision making when measured on MRI. Further research is needed to confirm these values in asymptomatic subjects and to establish the values in patients with patellofemoral instability.

Acknowledgments The authors would like to thank M. Holla, Orthopaedic surgeon, for providing the figures.

Conflict of interest The authors declare that they have no conflict of interest.

Open Access This article is distributed under the terms of the Creative Commons Attribution Noncommercial License which permits any noncommercial use, distribution, and reproduction in any medium, provided the original author(s) and source are credited.

\section{References}

1. Alemparte J, Ekdahl M, Burnier L, Hernández R, Cardemil A, Cielo R, Danilla S (2007) Patellofemoral evaluation with radiographs and computed tomography scans in 60 knees of asymptomatic subjects. Arthroscopy 23:170-177

2. Barnett AJ, Prentice M, Mandalia V, Wakeley CJ, Eldridge JDJ (2009) Patellar height measurement in trochlear dysplasia. Knee Surg Sports Traumatol Arthrosc 17:1412-1415

3. Biedert RM, Guhl C (1997) Axial computed tomography of the Patellofemoral joint with and without quadriceps contraction. Arch Orthop Trauma Surg 116:77-82

4. Biedert RM, Albrecht S (2006) The patellotrochlear index: a new index for assessing patellar height. Knee Surg Sports Traumatol Arthrosc 14:707-712

5. Bland JM, Altman DG (1986) Statistical methods for assessing agreement between two methods of clinical measurement. Lancet $1: 307-310$

6. Dejour H, Walch G, Nove-Josserand L, Guier C (1994) Factors of patellar instability: an anatomic radiographic study. Knee Surg Sports Traumatol Arthrosc 2:19-26
7. Goutallier D, Bernageau J, Lecudonnec B (1978) The measurement of the tibial tuberosity. Patella groove distanced technique and results. Rev Chir Orthop Reparatrice Appar Mot 64:423-428

8. van Huyssteen AL, Hendrix MRG, Barnett Al, Wakeley CJ, Eldridge JDJ (2006) Cartilage-bone mismatch in the dysplastic trochlea, an MRI study. J Bone Joint Surg 88-B:688-691

9. Inoue M, Shino K, Hirose H, Horibe S, Ono K (1988) Subluxation of the patella. Computed tomography analysis of patellofemoral congruence. J Bone Joint Surg Am 70:1331-1337

10. Laurin CA, Levesque HP, Dussault R, Labelle H, Peides JP (1978) The abnormal lateral patellofemoral angle: a diagnostic roentgenographic sign of recurrent patellar subluxation. J Bone Joint Surg Am 60:55-60

11. Miller TT, Staron RB, Feldman F (1996) Patellar height on sagittal MR imaging of the knee. Am J Roentgenol 167:339-341

12. Nomura E, Motoyasu M, Kurimura M (2003) Chondral and osteochondral injuries associated with acute patellar dislocation. Arthroscopy 19:717-721

13. Parker RD, Stefancin JJ (2007) First-time traumatic patellar dislocation: a systematic review. Clin Orthop Relat Res 455:93-101

14. Sasaki T, Yagi T (1986) Subluxation of the patella. Investigation by computed tomography. Int Orthop 10:115-120

15. Schoettle PB, Zanetti M, Seifert B, Pfirrmann CWA, Fucentese SF, Romero J (2006) The tibial tuberosity trochlear groove distance: a comparative study between CT and MRI scanning. Knee 13:26-31

16. Shin CS, Carpenter RD, Majumdar S, Ma CB (2009) Threedimensional in vivo patellofemoral kinematics and contact area of anterior cruciate ligament-deficient and -reconstructed subjects using magnetic resonance imaging. Arthroscopy 25:1214-1223

17. Staubli HU, dürrenhmatt U, Porcellini P, Rausching W (1999) Anatomy and surface geometry of the patellofemoral joint in the axial plane. J Bone joint Surg 81B:452-458

18. Staubli HU, bosshard C, Porcellini P, Rausching W (2002) Magnetic resonance imaging for the articular cartilage-bone mismatch. Clin Sports Med 21:417-433

19. Stanitski CL, Paletta GA Jr (1998) Articular cartilage injury with acute patellar dislocation in adolescents. Arthroscopic radiographic correlation. Am J Sports Med 26:52-55

20. Virolainen H, visuri T, Kuusela T (1993) Acute dislocation of the patella: MRI findings. Radiology 189:243-246 